\author{
Márgara Millán \\ Universidad Nacional Autónoma de México
}

\title{
Revistas y políticas de traducción del feminismo mexicano contemporáneo
}

\begin{abstract}
Resumen: En este ensayo me centraré en el análisis de tres importantes revistas y circuitos editoriales que constituyen, desde mi perspectiva, una parte importante de la política y la visibilidad feminista en el México contemporáneo: Debate Feminista, 1990, Fem, 1976 y La Correa Feminista, 1991. Voy a analizar estas publicaciones enfatizando lo que denominaré sus "políticas de traducción", aludiendo a los conceptos de feminismo y/o de género que promueven según las autoras y críticas que traducen. La noción de "traducción" significa, en este contexto, dos cosas: la traducción de ciertos autores y teorías y la manera en que estas traducciones se alinean con el contexto político local. Leeré estas traducciones entonces, de acuerdo a cómo se incorporan y participan de un panorama político más amplio, de cómo se presentan en un marco editorial determinado y del momento en que se hacen las publicaciones, entendiendo todo esto como maneras de intervenir en la arena política local. Al entender las traducciones como políticas de intervención, asumimos que esta es una de las formas en las que los grupos feministas establecen sus vínculos, sus alianzas, y sus plataformas para relacionarse con los movimientos sociales y los actores políticos nacionales. Me referiré al espacio de diálogo entre mujeres como un espacio problemático donde participan los grupos feministas y sus agendas, y las mujeres de diversos movimientos sociales y sus agendas. El texto de Rebecca $E$. Biron "Feminist periodicals and political crisis in Mexico"1 fue la inspiración central de este trabajo, así como el marco propuesto por Claudia de Lima Costa ${ }^{2}$ sobre transnacionalizacióntraducción como conceptos que necesitan ser clarificados desde sus significados locales y globales.
\end{abstract}

Palabras clave: revistas feministas; traducción; transnacionalización; feminismo mexicano.

Copyright (c) 2009 by Revista Estudos Feministas.

1 BIRON, 1996.

${ }^{2}$ COSTA, 2003 e 2006.

\section{La construcción de la polivalencia del sujeto del feminismo}

El feminismo, como corpus teórico-práctico, forma parte del paradigma ilustrado hoy en crisis. El ímpetu crítico, basado en la desnaturalización del hecho social, en el explosivo descubrimiento de que La ley ha sido enunciada, y por lo tanto, puede volver a serlo, informa y alimenta las 
utopías del siglo XX. La experiencia del mismo siglo, sin embargo, arroja una conciencia autorreflexiva que presenta los límites de esa "volibilidad" social, los límites de la concreción del "constructo" humano, los resultados sombríos de la razón moderna meramente instrumental. El feminismo, para ser comprendido cabalmente, debe ser analizado como parte de este paradigma en transformación, incluso, como una parte que contribuye a la desestabilización de ese paradigma, al enfatizar en algunos de sus desarrollos, la diferencia con el mundo guiado por el logos falocéntrico.

Es preciso señalar que la idea que del feminismo sostengo es una idea que lo refiere a un proceso en constante construcción, en la medida en que la "voluntad de saber" del feminismo se multiplica al hacerse visibles las diversidades del sujeto que lo constituye y sostiene. Al feminismo definido como la revelación de la contradicción centrada en la diferencia sexual y en el dominio masculino, le suceden formaciones prácticas y discursivas que obedecen más a la discusión y traducción de las diferencias entre las mujeres y sus contextos específicos. Esta deriva ha descentrado al propio discurso feminista, que hoy se reconoce a si mismo en la multiplicidad, es decir, como 'los feminismos'.

El sujeto del feminismo deviene complejo en tanto la conciencia de mujeres diversamente posicionadas en el todo social (donde ya interviene la dialéctica global/local), genera intervenciones culturales y políticas múltiples. El énfasis que pongo en el carácter polífono del feminismo contemporáneo no sólo recurre al reconocimiento de la multiplicidad (y contradictoriedad) de su sujeto (como sujeto de la enunciación), sino también a la diversidad de sus expresiones en términos de su práctica: arte, políica, filosofía, estilo de vida... En suma, el feminismo es considerado en este artículo como una fuerza preformativa de lo social, que por diversas vías, insiste en dar forma a la vida en común.

Ahora bien, ies pertinente hablar de 'feminismo mexicano'? Planteo que es de lo más importante comprender las formaciones discursivas y los tránsitos que componen los discursos locales. No sólo porque de esta manera el feminismo deja de ser un universal abstracto, sino porque el feminismo en tanto tal se encuentra siempre en diálogo y muchas veces en disputa con otros discursos críticos y con los procesos políticos relevantes de su contexto local.

La preocupación por el "feminismo mexicano" es válida, en el sentido de que quiere contextualizar las ideas y prácticas que en el discurso ilustrado aparecen como naturalmente "universales". El mundo global no significa desde esta perspectiva, la homogenización de los contenidos de las ideas y las teorías, sino sólo su circulación inevitable, para ser "traducida" o "trasladada" y articulada 
a los contextos locales, a las tradiciones de lucha que históricamente componen la densidad del territorio vivido. De esta forma, y aún considerando al feminismo como un fenómeno trasnacional, es de sustantividad política comprenderlo en su propia localización, en relación a lo nacional, y a la geopolítica colonial/poscolonial. Estudiarlo como parte de una cultura política específica, en diálogo y en ruptura con ésta. Es solo a través de esta localización de los feminismos nacionales que podemos descubrir las operaciones de traducción y diseminación del feminismo como fenómeno translocal, y elucidarlo como una fuerza política confrontada con otras.

La desconstrucción postestructuralista del sujeto atañe también al sujeto del feminismo. La construcción discursiva de La mujer como sujeto anclado sólo en la diferencia sexual da paso a la complejización identitaria producto de la interacción de cuerpo/cultura/raza/edad/ orientación sexual y otros vectores más de nuestras inserciones en lo social, y de lo cuál el concepto "género" quiere dar cuenta. Es por ello que la pregunta por lo "nacional" adquiere relevancia, ya que apunta hacia las contradicciones locales en procesos específicos de hegemonía cultural, así como a las relaciones globales de hegemonía/colonialidad. Esto ha sido señalado desde diversas localidades como la necesidad de construir un feminismo multirracial o multicultural. La comprensión del lugar de enunciación resulta entonces significativa para un dialogo feminista intercultural, que en todo caso, es también lo que está en juego en la traducción.

Las revistas y proyectos editoriales resultan en este sentido, para los viajes, traducciones, apropiaciones y resignificaciones de las teorías, espacios de mucha importancia, que se convierten en intervenciones políticas y culturales. Vinculan grupos, posibilitan la discusión pública de ciertos tópicos, y al mismo tiempo producen marcos de inteligibilidad de dichos tópicos.

Los feminismos son prácticas multilocalizadas, y sus revistas son especialmente relevantes en tender vasos comunicantes entre dichas prácticas. Al mismo tiempo, son instrumentos que ponen en contacto lo local con lo global. Las revistas feministas son espacios privilegiados que relevan la complejidad de las relaciones entre activismo, teoría, y políticas nacionales o locales. ${ }^{3}$ Esta relación entre teoría y política es también la relación entre el carácter trasnacional de diseminación de la teoría y los procesos locales de traducción-apropiación-reelaboración. Como lo plantea Claudia de Lima Costa ${ }^{4}$ existe una tensión entre las teorías metropolitanas y sus apropiaciones-traducciónes perifericas. En esta dinámica también se mueve la cuestión 
5 RICHARD, 2001, citada en COSTA, 2003. de si el Sur produce teoría, o es meramente fuente inspiradora para el Norte. En este esquema. Norte y Sur se reproducen como las fronteras internas entre la academia, los especialistas, los intelectuales y los movimientos sociales subalternos.

Nelly Richard ${ }^{5}$ llama la atención sobre el hecho de que la organización del fenómeno de la traducción se funda en un "aparato material-discursivo". Las revistas feministas son parte de dicho aparato que traduce y disemina teorías. $Y$ desde esta perspectiva, las revistas feministas son mediadores culturales.

En México el feminismo no ha entrado a la academia como en los Estados Unidos. No hay estudios de la mujer a nivel de pregrado; y es sólo a partir de la década de los noventa que los primeros espacios académicos formales aparecen. El Programa Interdisciplinario de Estudios de Género, PIEM, en el Colegio de México (1986), y el Programa Universitario de Estudios de Género, PUEG, de la UNAM (1992), son dos enclaves que poco a poco han ido desarrollando los estudios de género. Cabe mencionar también la especialización en estudios de género y de la mujer del Posgrado en Ciencias Sociales de la UAM-Xochimilco, también creada en los noventas. Las revistas feministas llegan a un público no muy amplio, y no son comúnmente parte de las bibliografías de la curricula en el área de ciencias sociales. Se encuentra más literatura feminista en el área de estudios literarios dentro de las Humanidades. Señalaremos enseguida una caracterización del feminismo mexicano en las últimas tres décadas y dentro de su trayectoria ubicaremos el lugar de las revistas más influyentes, dando cuenta de sus políticas de traducción en el escenario nacional.

\section{El feminismo como vanguardia: de la terapia a la política}

En los años setentas el mundo experimentaba el boom del feminismo. La revolución cultural de esa década, junto con la emergencia de las guerrillas en América Latina, fueron el referente para un feminismo militante, vanguardista y radical. La consciencia de la necesidad de la liberación femenina era, muchas veces, paralela a la consciencia de la necesidad de la liberación nacional. Sin embargo, los partidos políticos de izquierdas no reconocían la lucha feminista, considerándola pequeño burguesa.

El feminismo mexicano de los años setenta se conformó de manera muy clara como vanguardia: mujeres intelectuales radicales que buscaban la emancipación, que entendían al feminismo sobre todo como un cambio en sus propias vidas, que requerían de la autoconciencia generada en el pequeño grupo, y también de la acción pública, 
simbólica, ejemplar. La intervención política se consideraba absolutamente necesaria, pero problemática. Se daba bajo la forma de las manifestaciones simbólicas, de las discusiones y de las publicaciones. Se discutía en términos de cuál debía ser la relación entre feminismo y política.

Una revisión de las publicaciones de la época ilustra claramente este sentido militante de izquierda, del cual se alimentaba el feminismo mexicano, y el cual, sin embargo, fue desbordado rápidamente. La efervescencia de grupos feministas en esta década y sus discusiones internas y entre grupos muestran el crisol del movimiento; intelectuales, izquierdistas, autonomistas, anarquistas, institucionales, heterosexuales, lesbianas y homosexuales, eran definiciones identitarias que operaban en el feminismo mexicano en un diálogo con la teoría feminista, sobre todo anglosajona, y con la cultura política mexicana y sus referentes precisos: un Estado autoritario y su ejercicio del poder como represión y cooptación.

En esa década se sintetizó el espíritu libertario de los años sesenta, al tiempo que se problematizó la relación de la vanguardia feminista con la vanguardia política y con el "movimiento amplio" de mujeres. En el fondo, asistimos a la desestabilización de "la política" como una relación entre la vanguardia y las masas. En este proceso de autodefinición del feminismo, la necesidad de partir de la propia experiencia, de llevar lo personal al terreno de la política, fue algo que se resistió y contrapuso también a la necesidad del feminismo de actuar en términos partidarios, institucionales y vanguardistas.

Los Ilamados "grupos semilleros" mostraron las diferentes caras de un movimiento feminista que se debatía entre los principios, las rivalidades y la voluntad de incorporarse a las luchas amplias por la transformación políica del país. El feminismo se desarrollaba en muchos frentes: el pequeño grupo, la militancia partidaria, las luchas del movimiento sindical independiente, la academia, los medios de comunicación, el arte, la esfera institucional, etc. Se trataba de una multiplicidad de grupos y de personas que iban abriendo espacios, haciendo el arduo trabajo del topo que construye los puentes subterráneos que serán la base de las transformaciones culturales en el largo plazo.

Las principales publicaciones feministas de estos años son tres: La Revuelta, 1976, Cihuat, 1977 y Fem, 1976. Todas surgen tras el ambiente generado por la celebración oficial del Año Internacional de la Mujer, en 1975, misma que propició, por un lado, la apertura institucional hacia grupos de mujeres organizadas, y por otro, la activación de la organización de mujeres anti-oficialistas. Los grupos de mujeres feministas de esta época criticaban el autoritarismo 
y las relaciones jerárquicas de la izquierda tradicional, la subordinación de las demandas de las mujeres y de las mujeres mismas en los programas políticos y en las relaciones cotidianas de estas organizaciones. Todo ello parecía formar parte de la crítica al orden patriarcal, pero, al organizarse las feministas, muchas veces reproducían y formaban parte de una cultura de izquierda autoritaria y jerárquica.

El feminismo "histórico", como se le ha llamado al de estos años, llevaba a cabo acciones simbólicas emblemáticas, como protestar el 10 de mayo por la celebración del día de la madre, o en la celebración de Miss Universo, al tiempo que publicaba una serie de escritos fundantes de su horizonte teórico. El artículo de Marta Acevedo "Las mujeres luchan por su liberación. Nuestro sueño está en escarpado lugar", publicado en el suplemento cultural de la revista Siempre! en 1970, y que reseñaba el encuentro feminista de San Francisco de ese mismo año y señala el impacto que tiene el feminismo norteamericano sobre el mexicano.

Las tres revistas que hemos señalado son publicaciones de grupos constituidos, que encuentran en el acto editorial una práctica que los une, un espacio para dialogar internamente y un espacio para dialogar con otras mujeres. Se trata de pequeñas publicaciones, innovadoras incluso en su formato, que se lanzan como granadas en el movimiento social amplio.

Fem, la única de estas publicaciones que aún continúa publicándose, es fundada por Alaíde Foppa, guatemalteca, doctora en letras y crítica de arte, y la periodista y directora de Los Universitarios, Margarita García Flores. Ambas reúnen a un fuerte grupo de mujeres escritoras y creadoras. En su primer número aparecen Elena Poniatowska, Elena Urrutia, Margo Glantz, Nancy Cárdenas, Marta Lamas, entre otras, la crema y nata del feminismo mexicano de la época. Es la revista feminista más antigua de América Latina. Su formato como sus contenidos la define desde un inicio como una revista destinada a un público amplio, con una impronta poético-literaria, siempre con análisis de la cultura, cine, teatro, etc. Su políica editorial incluye escritos de autores varones, como Carlos Monsiváis, Tomás Mojarro, entre otros, ensayos reivindicativos de mujeres que forman la genealogía feminista, y pocas traducciones como artículos de fondo.

La Revuelta, por su parte, es producto de desconocidas jóvenas intelectuales y militantes, que desarrollan un feminismo radical que se ocupa desarrollar planteamientos teóricos desde el principio básico del feminismo: lo personal es político. Las reuniones del pequeño grupo daban el material para la escritura, y el periódico de La Revuelta se repartía a la salida de las fábricas. Se trataba 
también del esfuerzo de un feminismo de clase media ilustrada por salir de sí mismo y vincularse con las luchas sociales. El Colectivo la Revuelta deja de publicar su periódico en el número nueve, para iniciar la colaboración con el periódico Uno mas Uno hasta 1982.

Cihuat, por otra parte, era un órgano más político, el medio informativo de la Coalición de Mujeres Feministas, donde aparece de manera reiterada la denuncia de la situación de opresión y explotación de las mujeres mexicanas, así como la invitación a la lucha organizada. Su duración es de seis números, el último en 1978.

Fem vehicula un núcleo constante de crítica cultural latinoamericana. Sobretodo en sus primeros quince años, aglutina a un grupo amplio de voces femeninas intelectuales y creadoras. Se trata tanto por la duración de su publicación como por sus contenidos, de una visión amplia y ambiciosa del feminismo en América Latina. Polivalente, mantiene una clara definición política, sin afiliarse a un grupo específico, una preocupación teórica, sin encerrarse en un mundo académico, y una vinculación con autoras y movimientos del feminismo de su época. Presenta pocas traducciones, funcionando más a través de ensayos originales y mucho trabajo testimonial y de entrevista. Presenta, no obstante, textos de un feminismo que está marcando impronta en la crítica cultural, como su número de 1984 sobre las chicanas, con un texto de Cherrie Moraga, y en 1985 con un texto de Rosi Braidotti. En 1980 Alaíde Foppa es secuestrada en Guatemala, para nunca más ser vista con vida. Hasta 1986, la revista funciona a través de una dirección colectiva.

La interacción de lo global y lo local es clara en esta década. Muchos testimonios refieren el ímpetu organizativo que despertó entre las mujeres el hecho de que la primera de las cuatro conferencias de la mujer organizadas por la onu se cele-brara en la ciudad de México en 1975. Se abrían espacios por presiones diversas: los espacios oficiales y los contestatarios, todos con una bandera en disputa, la de las mujeres.

Los referentes del feminismo de los años setenta son por un lado una izquierda que no asumía el feminismo como parte central en su proyecto, sino como demanda "sectorial" o subordinada en la lucha estratégica y por el otro, un oficialismo autoritario que alineaba a las mujeres también como "sector" y además imponía su incorporación al "desarrollo nacional", asignándole sus tareas de género en la nación: la familia, la reproducción, la moral. Otro referente era un movimiento feminista internacional que impulsaba mucho las demandas de liberación sexual y de conocimiento y control del propio cuerpo, y urgía a las mujeres a criticar la ideología patriarcal. El movimiento lésbico-gay, 
parte del feminismo, es el otro referente, el cual finalmente se dibujó en estos años como un movimiento autónomo y en diálogo, muchas veces conflictivo, con el feminismo heterosexual.

El feminismo de esta época tiene la forma de una serie de grupos militantes y activistas que irradian contenidos a los movimientos sociales y a la sociedad en general. Esta fuerza es al mismo tiempo su límite. Esto tiene que ver con la cultura política en la cual se conforma el feminismo mexicano de los años setenta: el hecho de pertenecer a una clase media intelectual, de izquierda militante, que se organiza en grupúsculos de estudio y concientización, que busca intervenir en "las bases", convencer y guiar en el desarrollo de un movimiento social más o menos amplio. Hasta cierto punto, en este esquema, funciona la concepción de la vanguardia y las masas.

Algo que acompaña a este dispositivo de la izquierda de los años setenta y que tiene que ver más particularmente con la cultura políica mexicana es la difícil relación con el Estado. Un Estado autoritario cuyos mecanismos de afirmación como rector del sistema son la represión por un lado, la cooptación por el otro. Un sistema políico que se nutre del discurso revolucionario y populista, que hegemoniza el proceso de la Revolución mexicana para excluir a todo lo que se le opone. Un Estado que engulle o destruye.

El feminismo mexicano de los años setenta tiene que irse estableciendo como crítica a una izquierda que lo subordina, pero con la cual comparte muchos referentes e ideas; al mismo tiempo, tiene que decidir sobre la peligrosa relación con el Estado mexicano. Estas disyuntivas se delinean básicamente como la tendencia a permanecer fuera de los partidos políticos y del Estado, conformándose como movimiento independiente, contrario a cualquier oficialización o institucionalización. En torno a estas disyuntivas, los grupos militantes se dividen y rearticulan a lo largo de la década; su rearticulación se va haciendo en distintos frentes, lo cual vuelve al feminismo más polimorfo y abierto a más tendencias. De cualquier forma, y frente al autoritarismo del Estado mexicano, el feminismo así como la izquierda se definen como independientes. El feminismo se desarrolla mayormente fuera de los partidos de izquierda, quizá con la excepción del trotskismo, qué sí tuvo una política feminista en estos años. La relación con el Estado va a ser problemática, prefigurando el dilema de la siguiente década, entre institucionales y autónomas. Posteriormente esto se complejizará más, con la llegada de los fondos internacionales y las ONGs en los noventas. 


\section{El feminismo como política}

Es en estas tres décadas cuando el feminismo se conforma como una política que transforma las legislaciones vigentes, en los planos federal y estatal, y construye una estrategia de alianzas para su intervención pública. Particularmente en la década de 1980, lo que observamos es una enorme organización "micro" de las mujeres a través de la figura de las asociaciones civiles y las onG.

En la organización de las mujeres sindicalizadas, en contexto urbano y campesino, las mujeres adquirieron visibilidad mediante la estructuración de demandas específicas. En 1982 se celebró el Primer Encuentro Nacional de Mujeres en la ciudad de México y en 1986 el Primer Encuentro de Mujeres Campesinas de la CNPA, al igual que de la Coordinadora Plan de Ayala, ambas organizaciones independientes las más importantes de los indígenas campesinos. La Red Feminista Campesina se fundó en 1987, al igual que la Red de Promotoras Rurales. Mujeres en la educación, trabajadoras de la maquila, del servicio doméstico y del movimiento urbano popular, discutieron y articularon sus posiciones, mientras que el trabajo contra la violencia y por la salud se empezó a consolidar. El sujeto plural del feminismo se visibilizó a través de la organización de las mujeres en muchos frentes, al tiempo que la interacción entre clases y medios se potencializó y encontró un vehículo a través de las ONG.

Las estrategias de alianzas y participación política también se modificaron: el Movimiento Nacional de Mujeres le exigió al Estado participar en la atención de mujeres víctimas de violencia y se creó el Centro de Orientación y Apoyo a Personas Violadas (COAPEVI) en 1987. Así, fue abriendo el espacio de las mujeres organizadas en la institución pública, para desarrollar políticas hacia las mujeres. El feminismo transitó de posiciones vanguardistas

${ }^{6}$ El Salinismo se refiere al mandato del Presidente Carlos Salinas de Gortari quien llegó al poder tras unas elecciones muy contestadas en 1988 contendiendo con Cuauhtémoc Cárdenas en lo que se ha denunciado como un fraude electoral. El salinismo es la reconversión a las políticas neoliberales extremas con una política social adyacente, el neoliberalismo social según fue acuñado. PRONASOL fue el instrumento a cargo de las políticas sociales urbanas, y en el campo, sobre todo par alas mujeres, PROCAMPO y PROGRESA. a acciones de responsabilidad social, donde negoció espacios con el Estado, con lo cual la arena política del feminismo se amplió.

El Estado mexicano creó las Agencias Especializadas en Delitos Sexuales (AEDS) a partir de 1989 y el Centro de Apoyo a Víctimas de Violencia Sexual (ctA) en ese mismo año. En 1987 se creó la primera Secretaría de la Mujer en Guerrero. Y por supuesto, las mujeres son cada vez más objeto de las políticas sociales gubernamentales como Mujeres en Solidaridad del Programa Nacional de Solidaridad, PRONASOL, 1989, que era el programa oficial del Salinismo, ${ }^{6}$ y que fue el antecedente a la actual Secretaría de Desarrollo Social (SEDESOL). Sobre este hecho, Esperanza Tuñón afirma: 
${ }^{7}$ TUÑóN, 1997, p. 109.

${ }^{8}$ Sonia ALVAREZ, 1998
[...] no cabe duda de que las mujeres feministas se enfrentan hoy a la lógica propia de la política formal en el ámbito público - que difiere considerablemente de la dinámica particular desplegada con anterioridad por éstas en el campo de elaboración conceptual y cultural del feminismo y en los espacios politizados de la vida cotidiana - y, no sin resistencias, han aceptado participar o apoyar varios de los proyectos estatales, buscando, con ello, delimitar y profundizar su sentido. En esta postura opera, sin embargo, el aspecto anteriormente señalado de la dificultad política para ponderar los 'éxitos' del movimiento, ya sea como concesión genuina legitimada en la fuerza generada o como cooptación manipulada de las demandas feministas. ${ }^{7}$

La "Onegeización" de los movimientos sociales, ${ }^{8}$ y de la sociedad civil en general, se vislumbró con mayor claridad en la siguiente década, la de los noventa, pero sólo como fruto de lo iniciado en los años ochenta. A los temas de violencia y salud se incor-poraron los de pobreza, ciudadanía, equidad, atención legal y derechos civiles. Las políticas más articuladas del feminismo en torno a la denuncia y lucha contra la violencia se acompañaron de la campaña por una maternidad sin riesgos, aunada al eje de la democracia. Los años noventa fueron una eclosión en términos de las causas del movimiento: desde el uso de la lengua hasta los derechos multiculturales. El movimiento lésbico-gay, que también amplió su organización y visibilidad, inició estrategias para modificar la legislación vigente y así ampliar la definición de familia a familias, el derecho a la herencia, al matrimonio, ley que fue recientemente aprobada en la ciudad de México, en Noviembre del 2006.

La plataforma feminista de estos años muestra una complejidad creciente, donde las jornadas por la valorización del trabajo doméstico estuvieron acompañadas de campañas como Ganando Espacios, Acceso a la Justicia de las Mujeres, así como la fundación de la primera agrupación políitica feminista, DIVERSA, la cual pidió su registro electoral ${ }^{9}$.

La Ley de Asistencia y Prevención de la Violencia Familiar se instituyó en 1997, en 1999 se abrió el Instituto de la Mujer del gobierno de la ciudad de México y en el 2000 se promovió en el plano institucional la Primera Consulta Nacional por los Derechos de las Mujeres.

La política feminista se dibuja como un entramado complejo de acciones, demandas e intervenciones que representan a un sujeto social cada vez más diverso, capaz de estructurar demandas generales y específicas. La dimensión política del feminismo se muestra cada vez más como una intervención en el orden general de las cosas 


\begin{abstract}
${ }^{10}$ Sonia Alvarez asienta que en la década de los 90 s el feminismo latinoamericano "que otrora fue un movimiento relativamente aislado y restringido ... ahora puede caracterizarse más apropiadamente como un campo de acción expansivo, policéntrico y heterogéneo, pues abarca una amplia gama de terrenos culturales, sociales, políticos, gracias a la capacidad productiva de los feminismos, que ha extendido mucho su influencia cultural y política" (ALVAREZ, 1998, p. 93).
\end{abstract}

11 ALVAREZ, 1998. que rebasa el planteamiento sectorial y las demandas de igualdad. ${ }^{10}$

También parece establecerse una nueva relación entre la teoría y el movimiento, que supera nociones como las de "feminismo teórico" y "feminismo popular", para dar paso a una discusión más compleja dentro del propio feminismo, y de grupos feministas con otras corrientes. Todo esto tiene sus fundamentos en el "capital cultural y simbólico" que para las feministas mexicanas ha representado la incorporación masiva de las mujeres a la educación y al trabajo, así como la interacción de grupos de mujeres organizadas que desde los años ochenta han trabajado con mujeres pobres y marginadas.

La práctica feminista bajo las ONGs ha seguido dos tendencias: una dominada por el Centro, que trae el "desarrollo" a las áreas rurales y pobres, generalmente indígenas, y la otra, que intenta saltar las fronteras de clase y cultura o pertenencia étnica que separa a las mestizas de las indígenas, redefiniendo, hasta cierto punto, los fundamentos ilustrados del feminismo mestizo hegemónico. Mientras que la primera tendencia es al menos asimilacionista, por no decir imperialista, la segunda inicia reconociendo diferencias culturales, relativizando entonces la cultura hegemónica, y accediendo a una crítica de las formas capitalistas que se derivan de las formas culturales de las mujeres indígenas.

Paralelamente ocurría la discusión interna de los grupos feministas militantes, la cual giró en torno a la necesidad de la autonomía, frente a tendencias de participación institucional y a los financiamientos. La disyuntiva de "estar afuera o estar adentro" del sistema se dejó ver sobre todo en los encuentros y congresos feministas de los años noventa. Difícilmente se pudo lograr un acuerdo en relación con las políticas de alianzas, e incluso en términos de las prioridades del feminismo.

La "Ongeinización" del feminismo viene acompañando la transnacionalización del mismo, como lo analiza Sonia Alvarez. ${ }^{11}$ Para esta autora es claro que el campo de acción para el feminismo organizado en ONGs y que participa en las entidades y marcos institucionales, como los partidos políticos, el Estado, organismos e instituciones multilaterales, se ha ampliado de tal manera que es posible pensar en una agenda global de género; sin embargo, tras el boom de la "Ongeinización" del movimiento feminista se ha articulado un campo crítico en torno a cómo la agenda local de las feministas y, más centralmente, de los movimientos de mujeres, debe "caber" en los requerimientos de la agenda global a través, sobre todo, de las prioridades del financiamiento. Más aún, la distancia entre la existencia 
${ }^{12}$ ALVAREZ,: 1998, p. 93. de lo regional - global y el reconocimiento local se visibiliza. Esto permite afirmar que “... el campo feminista latinoamericano, cuyo alcance en la década de los noventa ha sido muy amplio, empieza a ser progresivamente mermado por relaciones desiguales de poder entre mujeres". ${ }^{12}$

\section{Las revistas de los años noventa}

En 1986 un grupo de mujeres feministas convocadas por Marta Lamas presentaban un proyecto al periódico La Jornada, con la intención de crear un suplemento que fuese un órgano de debate al interior del feminismo, y del feminismo con otras mujeres y con varones. El diario acoge el proyecto, pero, al estructurarse, hay diferencias entre un sector más periodístico y otro más intelectual. El Suplemento queda bajo la dirección de Sara Lovera, y mantiene un perfil informativo, mientras que en marzo de 1990 aparece Debate Feminista, bajo la dirección de Marta Lamas.

Debate Feminista es, con mucho, La revista teórica del feminismo mexicano. Dirigida por la antropóloga y activista Marta Lamas, continúa en colectivo con la labor que su directora ya ejercía antes: ser la principal traductora de la teoría feminista producida en inglés, francés e italiano. Ya en 1986, Lamas había publicado un importante artículo acompañando la traducción que presentaba la revista Nueva Antropología del influyente texto de Gayle Rubin, "The Traffic in Women: Notes on the 'Political Economy' of Sex", publicado en inglés 1975 en la compilación de Rayma Reiter, Toward an Anthropology of Women.

Debate Feminista tiene la forma de un libro de cerca de 300 páginas, con una periodicidad semestral, que presenta un estado del arte en cuanto a temáticas señaladas de manera monográfica. En sus 22 números publicados en estos diez años la revista consolida un perfil teórico, que pretende alimentar de reflexiones abiertas sobre ciertas problemáticas locales y trasnacionales: la democracia, la otredad, ley, cuerpo y sujeto, ciudades, escritura, políica, lo queer. Un continuo intento por ampliar el espectro del debate: impactar a través del feminismo la reflexión, ir más allá de él, tender puentes.

Debate Feminista ha sido la revista introductora en la academia y en las orientaciones del movimiento, de las principales autoras del feminismo contemporáneo: Teresa de Lauretis, Judith Butler, Adriana Cavarero, Lia Cigarini, Nancy Fraser, Julia Kristeva, Luce Irigaray. Presenta una estructura abierta, en el sentido de proponer siempre un espacio "Desde otro lugar", dedicado a miradas no declaradamente feministas y publicando autores varones en casi todos los números. 
Mantiene una estructura muy organizada pero flexible, que permite la aparición de secciones como "Desde el diván", confirmando el interés por la mirada psicoanalítica, algún texto que aparece "Desde la izquierda", "Desde lo cotidiano", o "Desde otro lugar", y que también abre un espacio para entrevistas, testimonios o denuncias, un espacio para fotografías, denominado "Desde la Mirada" y el Argüende, tradicional espacio recreativo a cargo de Jesúsa Rodríguez y Liliana Felipe, cabezas de un espacio crítico cultural lésbico muy importante. Todo esto acompaña al "núcleo duro" de la revista, que presenta textos organizados conforme a la temática de cada número.

Así, podríamos caracterizar Debate Feminista como una revista para el feminismo ilustrado, para un público especializado, fuertemente teórico, y en términos políticos de izquierda liberal, con un proyecto político institucional. El activismo de su directora, Marta Lamas, se ha centrado en torno a los derechos reproductivos y la despenalización del aborto.

Por su parte, La Correa Feminista, que se crea en 1991, estaría aglutinando a un feminismo radical y autónomo. Es editada por CICAM, Centro de Investigación y Capacitación de la Mujer. En su inicio, el objetivo es ser una especie de correa, como lo indica su nombre, entre los feminismos de los diferentes Estados del país, en un intento de sistematización del feminismo y para ir en contra de la centralización de la información en la capital.

En su recuento realizado en el número 19 de otoñoinvierno de 1998, plantean que en su definición inicial aparece señalada la problemática de quererse como un feminismo autónomo y radical, que reconoce las "necesidades sociales" de ampliar la democracia, frente a las necesidades del Estado de funcionalizar su proyecto neoliberal "incorporando aspectos de las demandas que el feminismo había desarrollado" (La Correa n. 19, otoñoinvierno 1998, 7 años de La Correa Feminista). Los primeros siete números de la revista son dedicados a tender puentes entre los Estados y el centro del país, algo así como la metrópoli y sus periferias, dado el carácter centralista y centralizado que conforma y permea a la estructura política y cultural mexicana.

Durante esta etapa se plantea como una correa informativa, explorando sobre todo la lucha contra la violencia hacia las mujeres y sus derechos humanos.

La Correa replantea su proyecto tras definir que el colectivo de las mujeres no es homogéneo, y contiene posiciones irreconciliables, afirmando que "[...] la voz de los sectores mayoritarios con mayor poder material y económico, tendía a la práctica de silenciar a las voces discrepantes". 
A partir del número 7 en 1994, la revista "se transforma en una revista de reflexión crítica, tras la búsqueda de elementos para un feminismo radical, rebelde, autónomo y antisistémico".

En su número 19 anuncia su forma virtual a través de Creatividad Feminista, en la internet. Las temáticas de los números subsecuentes presentaran textos centrados en el feminismo y la políica, la democracia, la guerra. La revista se articula con un sector del feminismo chileno, representado por la escritora Margarita Pisano. Ningún autor varón es publicado en La Correa.

Fem continúa durante esta década su puntual publicación, representando lo que Lamas Ilamaría "mujerismo", comprometiéndose con la escritura femenina, la entrevista, el recuento de los movimientos de mujeres, la literatura y la poesía de mujeres.

Para completar este panorama de fuerzas y feminismos, estaría el suplemento feminista de La Jornada, desde 1986 y hasta 1998, La Doble Jornada estará dirigida por Sara Lovera, y cumplirá un papel informativo y de difusión, un espacio para las redes de mujeres. A partir de 1998, el suplemento se vuelve La Triple Jornada, bajo la dirección de Ximena Bedregal y Rosa Rojas, del equipo de La Correa.

El objetivo de "fortalecer el debate respecto al papel de las mujeres en el mundo, que no es necesariamente adjudicarse la mitad del poder" ubica al suplemento como un posicionamiento al interior del feminismo, y cierra el círculo del debate que estableceremos acá.

Hay otras publicaciones feministas en México, notoriamente las del feminismo académico, que van apareciendo a la par que los nuevos departamentos de estudios de género o de las mujeres, como la Revista GenEros, de la Universidad de Colima, o La Ventana, de la Universidad de Guadalajara. Publicaciones como Nueva Antropología, Cuicuilco, Desacatos, Cuadernos Agrarios dedican algún número al feminismo y estudios de género, impactando cada vez más las diversas disciplinas. El referirse a este vasto espectro de estas publicaciones queda fuera de nuestro objetivo. Nos concentraremos en lo que hemos caracterizado como el feminismo hegemónico, que domina la escena desde el centro del país.

La transición de los setentas a los noventas está marcada por la eclosión de los movimientos sociales y la sociedad civil, la proliferación de ONGs que inician su trabajo con mujeres campesinas, populares e indígenas. La desestructuración del paradigma de la izquierda clásica, el fin del socialismo, de la guerra fría, de la guerrilla como forma de enfrentarse al poder. La redefinición de la relación con el Estado es un problema crucial. De hecho, con toda la 
institucionalidad. La preocupación por la democracia se acrecienta tras el proceso electoral de 1988. Se vuelve muy clara una parte de la izquierda que se define por el fortalecimiento de las instituciones democráticas, frente a otra que sigue pensando en la inviabilidad de éstas en un Estado donde domina la represión como la política de la economía neoliberal. La relación con lo institucional es uno de los vectores que más se politizan al interior de los movimientos sociales, incluido el feminismo. Paradójicamente, la creciente presencia de la sociedad civil organizada y sus conexiones con organismos trasnacionales permite que el feminismo se estabilice y se vuelva profesionalizante.

\section{Descentrando el feminismo: la politización del neozapatismo y la dificultad para tratar con lo indígena}

En el escenario político de inicios de los noventa en México, el salinismo había impregnado a amplias capas de la intelectualidad de izquierda con una cierta armonía frente a la globalización como inexorabilidad política y como futuro deseable. La crisis abierta por el levantamiento armado llama a cuentas definiciones que ya se pensaban zanjadas dentro de la cultura nacional: la violencia como recurso, la vigencia del Estado laico como norma, la preeminencia de la ley constitucional por encima de derechos de los pueblos. Las derivaciones del movimiento ponen en cuestión toda una idea de nación independiente y mestiza que nutre incluso los discursos críticos y de izquierda.

El movimiento armado zapatista abrió un espacio controversial para el feminismo mexicano: la articulación de las voces de las mujeres indígenas fue cuestionada por parte del movimiento feminista, sobre todo por la centralidad de la figura masculina del Subcomandante en el discurso en torno a las mujeres indígenas, mientras otras corrientes lo avalaban y reconocían. La misma forma de lucha del zapatismo provocó distintas reacciones y opiniones. El feminismo mexicano, a través de sus propias revistas, se articuló con posiciones más generales en torno al conflicto, aunque no podían dejar de reconocer la importancia de acciones emblemáticas del zapatismo indígena, como la ley revolucionaria de mujeres, la presencia de las comandantas y las palabras de las insurgentes. La difícil relación entre feminismo y zapatismo dejó ver terrenos no problematizados dentro del discurso crítico feminista: hasta dónde es tributario del discurso ilustrado de la modernidad occidentalizante en torno a lo indígena.

Esta discusión se desarrolló en los grupos de mujeres mestizas que trabajaban con mujeres indígenas desde la 
década de los ochenta, donde la difícil problemática de la interculturalidad se puso de relieve. A la luz de esta problemática, nociones como la nación liberal y los derechos individuales empezaron a ser relativizados. Apareció la noción de un feminismo indígena, y se acotaron los procesos específicos que produjeron distintas identidades. El feminismo dio paso a los feminismos. El "colectivo" mujeres se mostró como un proceso dialógico entre su propia heterogeneidad y la estructura social y cultural que lo contenía, del cual formaba parte y al cual transformaba o reafirmaba.

En esta sección enfatizaré las maneras en las que el "feminismo ilustrado" en sus distintas vertientes recibe e interpreta el levantamiento zapatista. La recepción y discusión que el zapatismo motivó es una dimensión importante de visibilización de las mujeres indígenas, y apunta a un posicionamiento dentro del feminismo nacional. Para analizar esta recepción, sólo me referiré a La Correa Feminista y a Debate Feminista, por ser las dos revistas que produjeron mayor discusión y problematización del movimiento zapatista y de la participación de las mujeres en él.

Ambas revistas acogieron críticamente la movilización zapatista, mientras que en Fem predominó la información solidaria del movimiento, y la denuncia a la situación de guerra. En Debate encontramos un posicionamiento a favor de los derechos reproductivos y, en consecuencia, un distanciamiento a lo que podría considerarse la influencia de corrientes eclesiales de base en la conducción del movimiento, tomando una prudente distancia frente al endiosamiento del mismo. Cabe señalar que esta posición se articuló al interior de la revista con ciertas traducciones (Norberto Bobbio y el Estado secular, por ejemplo), más que una discusión directa con el zapatismo; sin embargo esta discusión sí ocurrió en otro espacio editorial, la revista Proceso, al entablarse una polémica entre Marta Lamas y un grupo de pensadores (varones) que podemos afiliar a una corriente de la teología crítica, como veremos en detalle más adelante. La Correa, por su parte, mostró de manera más sistemática la gravedad de la situación de guerra, poniendo en tela de juicio los principios políticos que pueden desatar dinámicas de represión y desintegración de la vida cotidiana. Una denuncia cruda de la situación de guerra, donde no se le quita responsabilidad a los que poseen "la fuerza de la razón".

La primera compilación de materiales y posicionamiento es el que hace el grupo de La Correa Feminista, en la edición del primer tomo de Chiapas iY las mujeres

${ }^{13}$ La Correa Feminista, 1994. qué?, diciembre de $1994 .{ }^{13}$ Este grupo puede ser caracterizado como parte del "feminismo autónomo", es decir, aquél que se define como radical y crítico, en el sentido de 
14 "Para nosotras el feminismo es fundamentalmente pacifista $y$ antibélico [...] La guerra, en todas sus formas y expresiones ha sido instrumento vertebral del poder, del (des)orden y del dominio del sistema patriarcal..." (BEDREGAL, 1994, p. 43-44).

${ }^{15}$ BEDREGAL, 1994, p. 46.

${ }^{16}$ BEDREGAL, 1994, p. 46. permanecer fuera de las instituciones y desarrollar las posiciones del feminismo de la diferencia. Si bien la composición del primer tomo ya plantea la agenda del feminismo autónomo: la violencia contra las mujeres, la despenalización del aborto, la ausencia de las mujeres indígenas en el debate de las autonomías, es en la Introducción de Rosa Rojas y en el artículo de Ximena Bedregal titulado "Chiapas, reflexiones desde nuestro feminismo", donde el grupo editorial toma una posición crítica en torno al levantamiento insurgente.

Los puntos centrales se pueden sintetizar de la siguiente manera:

1. El feminismo es esencialmente pacifista. La guerra es parte del orden patriarcal. Por ello, el feminismo crítico debe tomar distancia de un proyecto que "libera" a través de la opción militar, ya que las estructuras militares son en sí mismas patriarcales, verticales y autoritarias. ${ }^{14} \mathrm{El}$ feminismo debe cuestionar la maquinaria de guerra y su lógica patriarcal, ilustrada por la guerrilla en Centro y Sudamérica. El que las mujeres se conviertan en soldados no debe ser visto como un logro.

Se hace la diferencia entre la critica a la guerra del feminismo y la que hace el liberalismo patriarcal. Este realiza una crítica hipócrita, porque reconoce la violencia en el otro, pero no en su propia lógica (la violencia del Estado de derecho, la paz inexistente, la desigualdad social y el exterminio). Frente a este posicionamiento liberal, la crítica feminista sería:

\begin{abstract}
la crítica profunda y radical de la base de estos delirios que con promesas y discursos de identidades recuperadas, de agravios vengados y de salvaciones definitivas solo nos presentan, desde los poderosos primero y desde los desposeídos después, [...] al aplazamiento eterno de la felicidad y donde - en aras de un supuesto bien superior - lo que siempre queda afuera es la libertad y la vida misma. ${ }^{15}$
\end{abstract}

El discurso y la práctica del EZLN "fortalece la idea de que a la violencia sólo se le puede combatir con la violencia y que ésta es válida si viene de los desamparados, los desposeídos, los oprimidos"; ${ }^{16}$ y actúa dentro de las leyes del sistema que critica al asumirse como ejército, declarar la guerra y acudir al reconocimiento de la Convención de Ginebra.

2. Reservas frente a la Ley Revolucionaria de Mujeres considerando que:

no es garantía de subversión del orden patriarcal que impera en las comunidades del territorio zapatista, en 
${ }^{17}$ La Correa Feminista, 1994.

${ }^{18}$ BEDREGAL, 1994, p. 46.
Chiapas y en el resto del país, ni será algo más que una parcial declaración de buenas intenciones, mientras las mujeres sigan siendo humanas de segunda clase impedidas por el autoritarismo masculino - que también las mujeres ayudamos a reproducir - de ser dueñas de sus cuerpos, con una maternidad libre y secundaria, mientras sus deseos de buena vida siga siendo tema secundario para algún futuro, mientras no sean material, política, social y simbólicamente dueñas reales de sus vidas, mientras su voz no sea elemento vertebral de la construcción social cotidiana. ${ }^{17}$

Y más adelante:

[...] en términos generales es evidente que no es feminista en la medida en que sólo plantea unas cuantas reivindicaciones para las mujeres y no una propuesta de comunidad desde la vivencia de lo femenino crítico y consciente, criticado y refundamentado... Desde nuestra perspectiva citadina, occidental e ilustrada, si las mujeres indígenas son invisibles en general y con la barrera de la guerra ya directamente inaccesibles, resulta prácticamente imposible saber si la ley es un producto real de un proceso de las mujeres frente a sus costumbres patriarcales y violentas o si es producto de los líderes ante la necesidad de incorporar a las mujeres a las tareas tradicionalmente masculinas y/o dar una idea de democracia más amplia $[\ldots] .{ }^{18}$

El principal punto ciego de esta crítica es el no ver a las mujeres indígenas como sujeto. La invisibilidad oscurece incluso su capacidad de agencia. ¿Son las mujeres las que enuncian la Ley o es una estrategia de la dirección masculina y patriarcal? Las mujeres quedan atrapadas en esa no visibilidad que las hace ser representadas únicamente como víctimas.

3. Reconocimiento de algunos aspectos del zapatismo:

Habría, sin embargo, ciertas analogías que acercan el "feminismo crítico" al movimiento rebelde. La primera es la analogía entre "indios" y "mujeres", ambos invisibilizados, marginales, que comparten el "no ser noticia". La segunda convergencia aparece en los aspectos "particulares" del discurso neozapatista, al desnudar la falacia que es el modelo neoliberal y sus promesas, y sobre todo, al reivindicar la validez de rebelarse, "y más que eso, ha instalado una esperanza para la diferencia, para la diversidad. Elementos que para las feministas deberían de ser alimento a su imaginación". ${ }^{19}$

Se valoran otras actitudes del EZLN, como el hecho de reconocer que se habla desde una localidad específica, 
${ }^{20}$ BEDREGAL, 1994, p. 46.

${ }^{21}$ ROJAS, 1995.
22 Originalmente la Consulta Nacional por la Paz y la Democracia consistía en cinco preguntas. A propuesta de Marcela Lagarde y Daniel Cazés se incluye una pregunta explícita sobre la necesidad de la paridad en la participación de las mujeres.

${ }^{23}$ OLIVERA BUSTAMENTE, 1995.

${ }^{24}$ BEDREGAL, 1995.

${ }^{25}$ OLIVERA BUSTAMENTE, 1995, p. 176.

${ }^{26}$ OLIVERA BUSTAMENTE, 1995, $\mathrm{p}$. 177. sin pretender imponer una "verdad" a todos, el acierto comunicativo presente en los comunicados tanto del CCRI (Comité Clandestino Revolucionario Indígena) como de Marcos, diciendo incluso en relación a esto que:

Desde otra lógica, y desde un orden simbólico que no es el del feminismo, nos ha dado una lección que debemos reconocer. Una comunicación parecida a esta ha sido una de las utopías feministas de la comunicación, que se ha perdido en la errónea creencia de que sólo podemos ser escuchadas si hablamos el lenguaje del otro $\left[\ldots . .{ }^{20}\right.$

El segundo tomo de Chiapas ¿y las mujeres qué? publicado en diciembre de $1995^{21}$ da cuenta del clima político y la gran movilización ciudadana que siguió a la incursión de febrero del ejército mexicano en la zona zapatista en busca del Subcomandante, que destruye una serie de comunidades y deja por un mes incomunicada a la zona. La gente se había refugiado en las montañas. Esta fue la segunda gran ofensiva tras los primeros doce días de guerra franca al estallar la rebelión. El movimiento de mujeres tuvo gran actividad. En febrero de 1995 se realizó la Primera Convención Nacional de Mujeres. Ya se había realizado la Convención Estatal de Mujeres, en Chiapas, en Julio de 1994. El ánimo en torno de un "nuevo pacto nacional" es el contexto para la petición de la sexta pregunta ${ }^{22}$ en la Consulta Nacional por la Paz y la Democracia del EZLN (que se efectuó el 27 de agosto de 1995).

Este tomo publica una interesante respuesta a las posturas principistas del feminismo autónomo, escrito por Mercedes Olivera Bustamente, "Práctica feminista en el movimiento zapatista de liberación nacional", ${ }^{23}$ acompañado del ensayo de Bedregal, "Un diálogo con Mercedes Olivera: Memoria y Utopía en la práctica feminista". ${ }^{24} \mathrm{El}$ primero es una visión desde Chiapas, a través del cuál se deja ver el entorno organizacional alrededor del zapatismo, y los avances dentro del zapatismo mismo, en relación a la participación de las mujeres indígenas y el reconocimiento de su voz y su trabajo. El avance que representa la movilización frente al modelo colonial impuesto a los y las indígenas. Para Olivera Bustamente, en el zapatismo y su contexto se juega "la posibilidad de convertir al feminismo en una práctica social amplia", ${ }^{25}$ y más adelante, "En suma, las feministas de campo, que hemos trabajado en Chiapas, valoramos los avances que han tenido las mujeres en la desconstrucción y reconstrucción de sus identidades de mujeres indígenas y campesinas pobres". ${ }^{26}$

Aparecen ya los tonos diferenciados del feminismo. Por un lado, urbano y radical, pero sectario y dogmático, 
27 OLIVERA BUSTAMENTE, 1995, $\mathrm{p}$. 184.

${ }^{28}$ BEDREGAL, 1995, p. 189c.

${ }^{29}$ Victoria SENDÓN y otras autoras, 1994.

30 SENDÓN apud BEDREGAL, 1995, p. 189d.

31 Alianzas entre diferentes ideologías y partidos, que consideran el reto del feminismo en México de los noventas, como lo señalan LAMAS et al., 1995. paradójicamente interesado en el feminismo de la diferencia, pero desinteresado de las diferencias mismas entre las mujeres. Claustro de la crítica radical al patriarcado. Por otra parte, el feminismo "de campo", mezclado con la cultura negada de la diversidad prohibida, la de las etnias, preocupado por las identidades. Este último, aunque reconozca las estructuras patriarcales, prioriza "la práctica de un feminismo amplio", fuera del claustro. Visibiliza a las mujeres indígenas como sujetos de agencia social.

En todo caso, ellas mismas (las mujeres indígenas) serán quienes decidirán impulsar o no el carácter feminista de sus organizaciones y de su movimiento. Nosotras las feministas, desde nuestro papel de asesoras o miembros de las ONGs ayudemos a que las mujeres se vean a sí mismas... ${ }^{27}$

Aun no se visibiliza la posibilidad de un feminismo indígena.

La respuesta de Bedregal reafirma la crítica al belicismo, aunque venga de los pobres, la idea de que y puntualiza en relación al escrito de Olivera, la negativa a subordinar el imaginario feminista, a jerarquizar prioridades.

\begin{abstract}
Mi feminismo... intenta ser una invitación a dar rienda suelta a la imaginación, a la autovalidación, a la crítica, a ser malas y saber que podemos ser peores, a no tener miedo, a tener memoria e historia, a sentirnos, a arriesgarnos, a darle nombre a lo que queremos, a inventar la libertad y otros mundos por encima de la(s) norma(s). A ser de-generadas, o sea a vivir fuera del género. $^{28}$
\end{abstract}

Pero como una no puede sino jerarquizar prioridades, la que se expone es la prioridad de una subjetividad radical y radicalizante, sobre otras maneras de construir agencia y autovalidación. Bedregal termina con una cita de Victoria Sendón: 29 "la fuerza del feminismo no radica en pensar en más y más cosas en nuestro saturado cerebro y en el mismo logos y en el mismo ethos, sino en pensar lo no pensado". ${ }^{30}$

El sector del feminismo en torno a la revista Debate Feminista es también intelectual, ilustrado, político y con alianzas amplias. ${ }^{31}$ Debate Feminista como grupo editorial expone su postura frente al movimiento en la editorial de su número 9, de marzo de 1994. "Cuando estalló la guerra en Chiapas, muchas nos preguntamos cuál era la perspectiva feminista sobre el conflicto" inicia y abre varias posiciones: las que su simpatía por el movimiento les cuestionaba su pacifismo, las que les concernía más la situación de las mujeres chiapanecas que eran desplazadas por el conflicto, las que se preocupaban más por el riesgo del proyecto democrático, las entusiastas por la Ley de Mujeres, las 
32 Chilango se le dice al que pertenece a la capital del país, Ciudad de México. La metrópoli capital es sin duda un lugar donde se centraliza mucho de la direccionalidad política del país.

${ }^{33}$ HERNÁNDEZ CASTILLO, p. 217.

preocupadas por la fuerza política de la iglesia católica. También mencionan que las que viajaron a Chiapas comprobaron, tras hablar con las feministas locales, que "una cosa es la mirada idealizadora chilanga, ${ }^{32}$ y otra la dura realidad social", la existencia de divisiones internas, el rechazo de muchas comunidades a la vía armada, actitudes mesiánicas, patriarcales y autoritarias de la Iglesia católica y de algunos miembros del EZ, hablan de mesianismo frente a "Ese otro camino [que] es el trabajo de masas..."

En este número Debate publica la Ley Revolucionaria de Mujeres y parte de la carta de Marcos donde se explica su proceso, un documento enviado por el grupo de San Cristóbal sobre los derechos reproductivos, y en relación a esto argumentan uno de los puntos centrales de su agenda política, el choque entre una concepción religiosa de la maternidad, donde el cuerpo de la mujer es considerado "instrumento divino", y donde "desde el momento de la fecundación, el ser humano en formación tiene plena autonomía de la madre [...]. Como ya lo señaló Juárez, las leyes no pueden basarse en creencias religiosas".

La revista publica de manera integra el "Documento pastoral sobre aborto" escrito por Don Samuel Ruíz, y el artículo de Michelangelo Bovero sobre el pensamiento laico.

En su número 24, de octubre del 2001, siete años después de la editorial de 1994, encontramos lo que podríamos denominar el fruto de la reflexión provocada por el levantamiento indígena para una parte del feminismo. Racismo y Mestizaje es el título de varios artículos que se preguntan sobre el racismo en México, su papel en la construcción de la nación. Artículos como el de Ruíz, "La india bonita: nación, raza y género en el México revolucionario", y el de Belausteguigoitia, "Descarados y deslenguadas: el cuerpo y la lengua en los umbrales de la nación", quieren indagar sobre el nacionalismo y la construcción de lo femenino indígena. El volumen reúne varios artículos sobre el neozapatismo, publica poesía tseltal y tzotzil, la intervención de la Comandanta Esther en el Congreso de la Unión y un buen testimonio fotográfico.

El artículo de Aída Hernández "Entre el etnocentrismo feminista y el esencialismo étnico. Las mujeres indígenas y sus demandas de género" es un ensayo crítico al feminismo mexicano por su etnocentrismo. Las mujeres indígenas se verían en medio de "un movimiento indígena que se niega a reconocer su sexismo y un movimiento feminista que se niega a reconocer su etnocentrismo". ${ }^{33}$

El punto, para esta autora, es la articulación que hace un grupo de mujeres indígenas de las demandas de género con las demandas autonómicas de sus pueblos, como una "Iucha en muchos frentes", ante la cuál el "feminismo 
34 HERNÁNDEZ CASTILLO, p 207, nota a pie n. 4.

${ }^{35}$ LAMAS, 1994, p. 141

${ }^{36}$ LAMAS, 1994, p. 141.

${ }^{37}$ ROJAS, 1994 , p. 145 hegemónico" no tiende puentes. Y define por feminismo hegemónico al "surgido en el centro del país, y teorizado desde la academia en el que la lucha contra el aborto y por los derechos reproductivos ha sido central". ${ }^{34}$ En realidad, el "centramiento" de este feminismo hegemónico le impide también tender puentes con sectores de religiosas que desde hace tiempo hacen una labor de reflexión y organización de las mujeres sobre su condición. El resultado es una agenda feminista hegemónica excluyente, que privilegia las demandas de la experiencia urbana ilustrada, y adscrita a una noción de derechos individuales que no atiende a la idea - quizá para siempre perdida - de comunalidad.

Marta Lamas y el Subcomandante Marcos habían intercambiado misivas en relación a la despenalización del aborto, en La Jornada, el 29 de abril y 11 de mayo de 1994: La discusión se da por una supuesta demanda del EZLN a que en la reforma al Código Penal en Chiapas se instituya la penalización del aborto. Lamas establece el punto de que la despenalización del aborto es una cuestión central en un proyecto verdaderamente democrático, en el sentido de "el respeto a la pluralidad y las garantías individuales". ${ }^{35}$ Pero también aclara que en nuestros países las mujeres con recursos económicos pueden tener abortos sanitarios, mientras que las mujeres pobres recurren a interrupciones del embarazo que las colocan en alto riesgo de muerte. Es decir, las "garantías individuales" están acotadas por la pertenencia a la clase social. Por último establece muy bien el punto de que la no despenalización del aborto en nuestro país habla de "la dificultad del estado mexicano para hacer valer la separación entre estado laico e iglesias". ${ }^{36}$

El 11 de mayo de 1994 Marcos niega que el EZLN esté demandando la penalización del aborto, ni la reformulación el código penal, y transcribe el punto 27 de las demandas del EZLN donde lo que se pide es "Que se quite el Código Penal del Estado de Chiapas porque no nos deja organizarnos más que con las armas [...]".

En su respuesta, Marcos enuncia un tropos que se repite en varios comunicados. La idea de que la Ley de Mujeres fue impuesta por las mujeres zapatistas al EZLN, la idea de que los cambios que están haciendo las mujeres son "a pesar de periódicos, iglesias, códigos penales y nuestra, justo es reconocerlo, resistencia como varones a ser arrojados del cómodo espacio de dominación que nos heredaron". ${ }^{37}$

Finalmente, en el estilo posdata, hay dos fuertes aseveraciones: que las indígenas abortan y no por elección propia, sino por desnutrición crónica, y que no piden clínicas para abortar porque no tienen clínicas de partos, y que 
${ }^{38}$ ROJAS, 1994, p. 145.

${ }^{39}$ LAMAS, 2003, p. 59.

${ }^{40}$ SICILIA, 2003, p. 59.

${ }^{41}$ SICILIA, 2003, p. 58. "subir las lomas cargando un tercio de leña es algo que ningún código penal toma en cuenta [...]". ${ }^{38}$

Esta polémica toma forma de nuevo años más tarde, de diciembre del 2002 a enero del 2003, del número 1362 al 1367 de la revista Proceso. Javier Sicilia responde a un artículo de Monsivais titulado "De Obispos y de geología social" (Proceso 1362) ocasionado por la "recomendación" de los obispos motivada ante la Primera Cumbre de Mujeres Indígenas de las Américas, en diciembre del 2002. Participan en la polémica entre otros Gustavo Esteva y Sylvia Marcos. Todos comparten la cercanía teórica con Ivan Illich, y su importante obra sobre el género vernáculo y el sexismo moderno.

¿Qué es lo que se dirime en esta polémica? Se discute en torno a los derechos reproductivos y la despenalización del aborto. Para Lamas,

[...] en este debate sobre el género, que es también sobre el esencialismo, sería interesante entrar a definir los contornos de ese mundo justo y libre que creemos posible, que para mi no es el del pasado ni el del presente. Un mundo que reconozca la diferencia sexual sin imponer falsas complementariedades y que favorezca el desarrollo de las potencialidades humanas...en una utopía de un mundo sin explotación económica los derechos sexuales y reproductivos son un eje fundamental. ${ }^{39}$

Sicilia responde: "Como usted puede ver, no creo en los derechos sexuales ni reproductivos en ningún tipo de sociedad. Creo en la proporción, en la persona, en la diferencia, en el deber y en el lugar del misterio". ${ }^{40}$

Desde una crítica cristiana a la modernidad, Sicilia plantea al género como un ordenamiento vernáculo, versus el ordenamiento moderno, donde "el derecho romano es la única medida de todo". Lo vernáculo, ordena de manera proporcional el universo humano, mediante una guía que modera la acción del hombre frente a la naturaleza física y humana. "Esta proporción implica, entre muchas otras cosas, entender que la maternidad es un don, no un derecho [...]." 41

Para Sicilia, la pérdida de esa proporcionalidad ocurre en el desarrollo societal que se centra en lo económico como valor absoluto, "donde el ser humano ha desalojado de su vida el orden de lo sagrado", y es justamente ahí donde se genera el debate moderno de los derechos reproductivos.

Esteva, desde otro lugar, responde a Lamas con la distinción propuesta por Illich entre patriarcado:

para hablar de la dominación de los hombres sobre las mujeres en las condiciones vernáculas" y sexismo, 
42 ESTEVA, 2003, p. 60.

${ }^{43}$ María Estela JOCÓN-MAYA apud ESTEVA, 2003, p. 81.

${ }^{44}$ Sylvia MARCOS apud ESTEVA, 2003, p. 80

${ }^{45}$ SICILIA, 2003, p. 59

${ }^{46}$ ESTEVA, 2003, p. 61.

47 HERNÁNDEZ CASTILLO, 2001. "para hablar de las consecuencias de la desventaja que sólo una sociedad ideológicamente igualitaria puede imponer a sus sujetos humanos que diagnostica como pertenecientes al sexo femenino [...] sin el cuál una sociedad basada en la mercancía no podría existir... ${ }^{42}$

Proporcionalidad y diferencia, igualdad y capitalismo serían los pares que sostienen este emplazamiento. Sylvia Marcos, interviene para señalar la importancia de escuchar lo que están diciendo las mujeres indígenas, desde su localización espiritual y práctica. María Estela JocónMaya, de Guatemala, aclara: "Se entiende por la práctica del enfoque de género una relación respetuosa... de balance, de equilibrio - lo que en Occidente sería la equidad". ${ }^{43}$

La postura de Lamas es caracterizada como colonialista desde las tres perspectivas, y por motivos diferentes. Para Sylvia Marcos, "La preocupación es que el discurso feminista, colocado en la élite urbana, actúe como elemento colonizador e "involuntariamente" hegemónico..."44

Para Sicilia, [las mujeres]

tienen todo el derecho de defender... sus derechos reproductivos y aplicarlos en sus cuerpos, lo que no tienen derecho es a erigirlos como un valor supremo de la mujer (por eso he dicho que su discurso [el de Lamas] es colonialista, pretende hacer decir a las mujeres indígenas lo que nunca dijeron... ${ }^{45}$

Y para Esteva, "no hay alguna noción que flote por encima de todas las culturas y épocas, como pretenden quienes comparten la mentalidad económica de la modernidad, contra cuyo aliento colonialista necesitamos luchar". ${ }^{46}$

En el fondo, lo que se pone en juego son diversos esencialismos: el de Lamas al poner como central y universal las demandas de las mujeres urbanas por los derechos sexuales y reproductivos; el de Sicilia y Esteva, al negarle a la modernidad cualquier cualidad, verla como totalmente dominada por la centralidad económica, sin resquicios ni resistencias. Mientras el pasado para Esteva y Sicilia es siempre mejor, para Lamas no es sino ausencia de derechos. Para Lamas no hay nada en el pasado que deba ser recuperado, para Sicilia lo hay todo. Rotos los vasos comunicantes, la polémica no transita al diálogo.

En medio de la polarizada discusión, Sylvia Marcos llama la atención hacia las declaraciones de las mujeres indígenas de la Cumbre, diciendo: hay que oírlas con cuidado, traducirlas. Como afirma, ${ }^{47}$ las mujeres indígenas quedan entre el etnocentrismo feminista, donde el "etnos" 
viene de la hegemonía cultural, la pertenencia a la clase dominante, y la adherencia al modelo modernizador, y el esencialismo étnico, que viene de la prevalencia de la comunidad ya sea cristiana o indígena, como asentamiento de alguna manera fijo e inmutable, o en el mejor de los casos, inspirador para la reconstrucción comunitaria.

En todo caso, la ausencia de las voces de las mujeres indígenas, o la poca referencia a ellas en esta polémica, acusa la segregación clasista y étnica del nacionalismo/ indigenismo mexicano que ha creado grandes separacines entre las mujeres. Estas separaciones hoy están redefiniédose, y el feminismo en algunas de sus vertientes se encuentra desarrollando posturas y miradas sobre las mujeres indígenas fuera del indigenismo prevaleciente, es decir, reconstructor de una relación de colonialidad paternalista (o maternalista) hacia el mundo indígena. Ello implica una serie de desplazamientos dentro del aparato crítico feminista, que, como condición para aperturar a la diversidad de lo femenino y del sujeto del feminismo, debe decolonizar sus propias asunciones.

Por otra parte, la dinámica local-global también incide en el posicionamiento del "feminismo indígena" emergente. Las voces de las mujeres indígenas están ya en otros contextos, los que el movimiento local e internacional ha ido abriendo, campos de enunciación para una palabra propia. Y por "propia" no quiero decir intocada por discursos varios. Justamente lo contrario, palabra que se a/ propia de una multiplicidad de discursos para "ser en el mundo", y "nacer el mundo", llena de traducciones que han significado ciertas traiciónes, para recordar a Anzaldúa.

La discusión convoca a elucidar localmente los significados específicos de la "emancipación" de las mujeres indígenas, desestabilizando nociones unívocas y universalistas de conceptos como "liberación femenina". No abdicar de estos conceptos, sino llenarlos de contenido diverso, localizado y útil para un sujeto concreto, ya que las realidades son múltiples. En conjunto, el zapatismo indígena mostró al feminismo hegemónico mexicano una visión de mujeres indígenas menos apegadas al silencio y a la sombra, más actoras políticas, sujetos plenos y diferenciales, conscientes de su discriminación social, racial y de género, y conscientes también, de sus diferencias identitarias. Su discurso es cada vez más audible, y en él combinan reclamos por justicia social y genérica y por reconocimiento cultural.

La discusión en torno al zapatismo también provocó en el feminismo hegemónico una mayor autorreflexión en el espejo clasista y racista de la nación. La nación aparece como objeto de elucidación feminista, que no se agota en su caracterización en tanto "patriarcal", sino en la recons- 
${ }^{48}$ Hay ejemplos alentadores de estas alianzas, uno de ellos fue el encuentro-taller realizado en la Cd de México en noviembre del 2005 con mujeres indígenas de México, Guatemala y Nicaragua convocado por el CIESAS a través de Aída Hernández. El libro La doble mirada, 2005, coordinado por Martha Sánchez Néstor, Amuzga de Guerrero y promovido por Aída Hernández, Teresa Sierra y Olivia Gall, se presentó en ese contexto. Otro libro importante es el de Zoila Reyes Hernández, Sólo soy una mujer, 2005, editado por Gisela Espinoza Damián, Universidad Autónoma Benito Juárez de Oaxaca. trucción del complejo dialogismo que la constituye, y del cuál las mujeres, blancas y de color, formamos parte. Y quizá lo más importante, en este diálogo intercultural, el feminismo hegemónico voltea a verse a sí mismo, y a expandir, en algunos casos, las fronteras que su propia localización le impone, empieza a ver más, y al hacerlo, avanza hacia una convivencialidad más inclusiva y hacia una voz más plural.

Podemos afirmar que estamos en otro momento a nivel nacional: para el feminismo mexicano, la interlocución y el diálogo con las mujeres indígenas lo ha obligado a revisar su etnocentrismo y por ende su agenda política. Para las mujeres indígenas, el diálogo con las feministas ha contribuido para el esclarecimiento de su propio feminismo indígena y su propia agenda política. Las instituciones académicas son aún muy rígidas y poco creativas frente al reto de abrir espacios para dialogar con inteligencias y sabidurías que corren por caminos no formales. ${ }^{48}$ Sin embargo, el trabajo conjunto que están realizando mujeres mestizas e indígenas en torno a los derechos de las mujeres, las autonomías de los pueblos indios y el Estado multicultural es ya un referente insoslayable que mostrará su impacto en los frutos futuros del feminismo mexicano.

En cuanto a las revistas analizadas, podemos afirmar que las traducciones y discusiones que apenas adquieren visibilidad son las referentes al racismo y etnocentrismo que informa la cultura nacional. El poco impacto que el feminismo chicano tiene en el feminismo hegemónico da cuenta de esto. No obstante, la situación nacional ha sufrido un desquebrajamiento. La manera en que las mujeres indígenas articulan dos de sus realidades sustanciales, los pueblos y el género, apenas inicia un ciclo de reflexividad, donde el feminismo como teoría crítica se descentra de la diferencia sexual para articularla a las otras diferencias múltiples de la colonialidad.

\section{Referencias bibliográficas}

ALVAREZ, Sonia E. "Los feminismos latinoamericanos se globalizan en los noventa: retos para un nuevo milenio". In: TARRÉS, María Luisa (Ed.). Género y cultura en América Latina. México: El Colegio de México, 1998. p. 89-136.

BEDREGAL, Ximena. "Chiapas, reflexiones desde nuestro feminismo". La Correa Feminista, n. 1, v. 1, 1994.

"Memoria y utopía en el movimiento feminista. Un diálogo con Mercedes Olivera". In: ROJAS, Rosa (Ed.). En Chiapas ¿y las mujeres qué? México: CICAM, 1995. v. II. p.185-189. 
BIRON, Rebecca E. "Feminist Periodicals and Political Crisis in Mexico: Fem, Debate Feminista and La Correa Feminista in the 1990's." Feminist Studies, v. 22, n. 1, 1996. p. 151-169.

COSTA, Claudia de Lima. "As publicacões feministas e a política transnacional da traducão: reflexões do campo". Revista Estudos Feministas, v. 11, n. 1, p. 254-264, 2003. . "Lost (and Found?) in Translation: Feminisms in Hemispheric Dialogue." Latino Studies, v. 4, n. 1, 2006. p. 62-78.

ESTEVA, Gustavo. "Racismo y sexismo: dos caras de la ceguera dominante". Proceso, n. 1367, p. 60-61, 12 jan. 2003.

HERNÁNDEZ CASTILLO, Rosalva Aída. "Entre el etnocentrismo feminista y el esencialismo étnico. Las mujeres indígenas y sus demandas de género". Debate Feminista , n. 24, p. 206-229, oct. 2001.

LA CORREA FEMINISTA. México, n. 1, v. 1, 1994.

LAMAS, Marta. "El EZLN, el Vaticano. El aborto y el Estado mexicano". La Jornada, 29 Apr. 1994. Rpt. In: ROJAS, Rosa (Ed.). En Chiapas ¿y las mujeres qué? México: CICAM, 1994. v. I. p. 139-142

"Género: ibienvenido un debate tan necesario!" Proceso, n. 1366, p. 58-59, 5 jan. 2003.

LAMAS, Marta, MARTíNEZ, Alicia, TARRÉS, María Luisa, and TUÑÓN, Esperanza. "Building Bridges: The Growth of Popular Feminism in Mexico." BASU, Amrita (ed.). The Challenges of Local Feminisms: Women's Movements in Global Perspective. Boulder: Westview Press, 1995. p. 324-351.

OLIVERA BUSTAMANTE, Mercedes. "Práctica feminista en el movimiento zapatista de liberación nacional". In: ROJAS, Rosa (Ed.). En Chiapas ¿y las mujeres qué? México: CICAM, 1995. v. II. p. 168-184.

ROJAS, Rosa (Ed.). En Chiapas ¿y las mujeres qué? México: CICAM, 1994. v. I.

En Chiapas ¿y las mujeres qué? México: CICAM, 1995. v. II.

SENDÓN, Victoria; SÁNCHEZ, María; GUTíN, Montserrat; APARICl, Elvira. Feminismo holístico, de la realidad a lo real. Barcelona: Cuadernos del Agora, 1994.

SICILIA, Javier. "La miseria de los derechos reproductivos". Proceso, n. 1367, p. 58-59, 12 jan. 2003.

TUÑóN, Esperanza. Mujeres en escena: de la tramoya al protagonismo (1982-1994). Mexico: UNAM/Joaquín Mortiz, 1997.

[Recebido em setembro de 2009

e aceito para publicação em outubro de 2009] 
Journals and Translation Policies of Contemporary Mexican Feminism

Abstract: This essay focuses on the analysis of three important journals and editorial circuits which constitute, from this perspective, a significant part of feminist policy and visibility in contemporary Mexico: Debate Feminista, 1990, Fem, 1976 and La Correa Feminista, 1991. My purpose is to analyze these publications emphasizing their "translations policies", mentioning the concepts of feminism and/or gender they promote through the authors and the criticism they choose to translate. "Translation", in this context, means the translation of certain authors and theories and the way such translations fit into the local political context. I will read these translations according to the way they are incorporated into, and participate in, a broader political scenario; the way they present themselves in a certain editorial picture and the historical moment they are published, understanding them as ways of intervening in the local political arena. Understanding the translations as intervention policies, we assume that this is one of the ways through which feminist groups set up connections, alliances and plafforms to establish a relationship with both the social movements and national political actors. I will refer to the dialogical space between women as a problematic arena of feminist groups and their agendas, and women from several social movements and their agendas. Rebecca E. Biron's text "Feminist Periodicals in Mexico" (1996) was the core inspiration for this work, as well as the argument proposed by Claudia de Lima Costa $(2003 ; 2006)$ on transnationalization-translation as concepts that need to be clarified from their local and global perspectives.

Key Words: Feminist Journals; Translation; Transnationalization; Mexican Feminism. 